Supplementary Material

\title{
Unusual Reduction of Graphene oxide by Titanium dioxide electrons Produced by Ionizing radiation. Reaction Products and Mechanism
}

David Behar ${ }^{1}$, Tijana Rajh², Yuzi Liu², Justin Connell ${ }^{2}$, Vojislav Stamenkovic ${ }^{2}$ and Joseph $\operatorname{Rabani}^{1 *}$

${ }^{1}$ Institute of Chemistry, The Andy Brickman Memorial Accelerator bldg, The Hebrew University of Jerusalem, Edmond J. Safra Campus, Jerusalem 91904, Israel ${ }^{2}$ Argonne National Laboratory, 900 South Cass Avenue, Bldg. 440, Argonne, IL 60439, USA

*Corresponding author: rabani@mail.huji.ac.il

Raman spectra of GO-rGO solutions at different stages of GO reduction by $\mathrm{e}_{\mathrm{TiO} 2}{ }^{-}$are presented in Fig. S1. The peaks intensities were normalized to the intensity of $1330 \mathrm{~cm}^{-1}$. The maxima at $\sim 1330$ and $\sim 1600 \mathrm{~cm}^{-1}$ are assigned to the well-known D and G peaks, respectively, both dominated by the $\mathrm{sp}^{2}$ phase, as excitation resonates with $\pi$ states. ${ }^{1}$ In a nutshell, the $\mathrm{G}$ peak is associated with the bond stretching of $\mathrm{sp}^{2}$ atoms in both rings and chains, while the $\mathrm{D}$ peak is associated with the breathing modes of $\mathrm{sp}^{2}$ atoms in rings, but requires $\mathrm{sp}^{3}$ defects to be activated. ${ }^{2}$ The $2 \mathrm{D}$ peak associated with overtone of TO phonons in GO and $\mathrm{rGO}\left(\sim 2700 \mathrm{~cm}^{-1}\right)$ remains very week suggesting introduction of $\mathrm{sp}^{3}$ defect like structures rather than reestablishing of graphitic structures upon reduction of $\mathrm{GO} .^{3}$ 


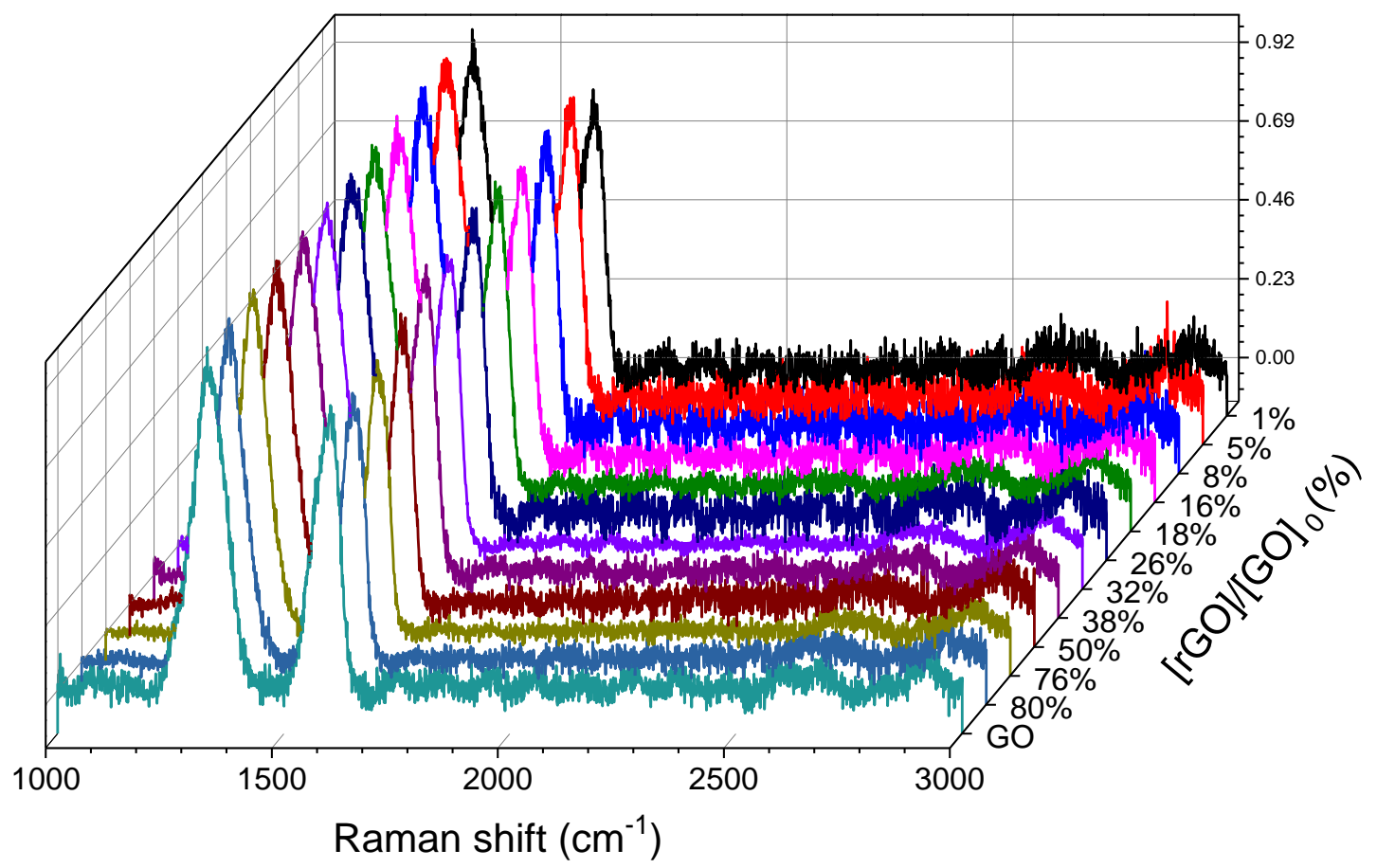

Figure S1. Development of normalized Raman spectra upon reduction of GO to rGO The reduced GO expressed in terms of percent $\left(100 x[\mathrm{rGO}] /[\mathrm{GO}]_{0}\right)$.

The much larger cross section of interaction of $\mathrm{sp}^{2}$ phase compared to $\mathrm{sp}^{3}$ (50-250 times) makes it difficult to quantitatively analyze the concentration of the $\mathrm{sp}^{3}$-type defects. The ratio of intensities $\mathrm{I}(\mathrm{D}) / \mathrm{I}(\mathrm{G})$ is typically used to observe qualitative changes in $\mathrm{sp}^{2}$ induced by introduction of $\mathrm{sp}^{3}$ defects. ${ }^{4}$ The $\mathrm{I}(\mathrm{D}) / \mathrm{I}(\mathrm{G})$ ratio in nanoscale structures initially sharply decreases upon introduction of a topological disorder into the graphene-like structures. The bonding remains mainly $\mathrm{sp}^{2}$, but the weaker bonds affect the vibrational modes. At low $[\mathrm{rGO}] /[\mathrm{GO}]_{0}$ fractions (red curve, Fig. S2) there is an initial sharp decrease of $\mathrm{I}(\mathrm{D}) / \mathrm{I}(\mathrm{G})$. This is followed by conversion of $\mathrm{sp}^{2}$ to $\mathrm{sp}^{3}$ sites and the consequent change of $\mathrm{sp}^{2}$ configuration from rings to 
chains, which is associated with increasing $\mathrm{I}(\mathrm{D}) / \mathrm{I}(\mathrm{G})$ (Fig. S2, blue curve). This process is usually accompanied with a shift of the $G$ peak, due to the confinement of $\pi$ electrons in shorter chains. In Fig. S3 a decrease in the intensity of the low energy part of the G peak and overall shift of intensity toward $1600 \mathrm{~cm}^{-1}$ is observed above $50 \%$ conversion of GO to rGO.

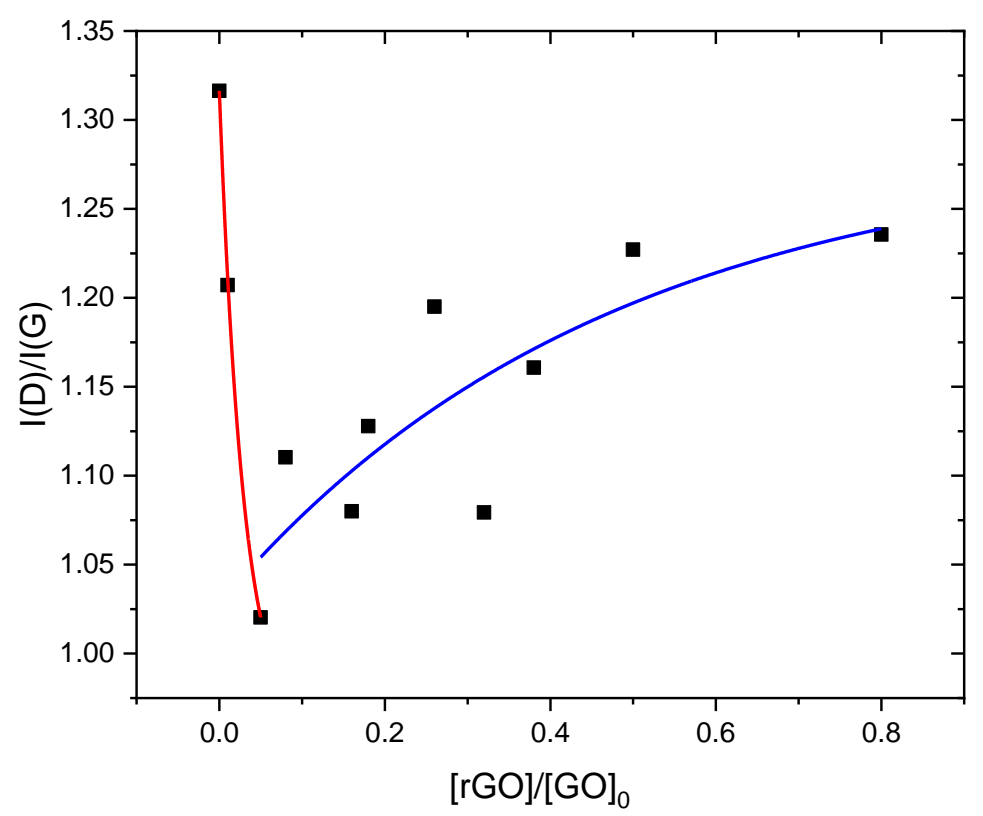

Figure S2. Variation of $\mathrm{I}(\mathrm{D}) / \mathrm{I}(\mathrm{G})$ as the reduction of $\mathrm{GO}$ proceeds. This is in agreement with

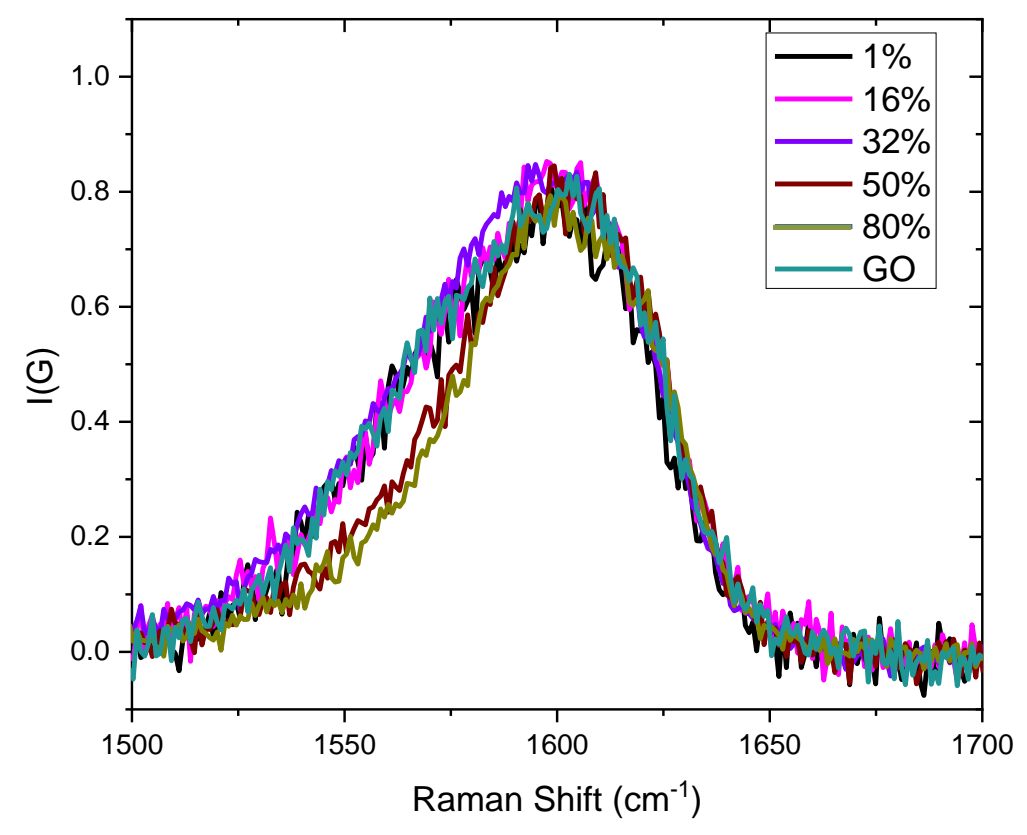

earlier studies of transformation of graphitic like structures upon introduction of $\mathrm{sp}^{3}$ defects. $^{2,4}$ 
Figure S3. Development of G peak shape of the Raman spectra upon reduction of GO to rGO (expressed in terms of percent $\left(100 x[\mathrm{rGO}] /[\mathrm{GO}]_{0}\right)$ )

1. Wu, J. B.; Lin, M. L.; Cong, X.; Liu, H. N.; Tan, P. H., Raman Spectroscopy of Graphene-Based Materials and Its Applications in Related Devices. Chemical Society Reviews 2018, 47, 1822-1873.

2. Ferrari, A. C.; Robertson, J., Raman Spectroscopy of Amorphous, Nanostructured, Diamond-Like Carbon, and Nanodiamond. Philosophical Transactions of the Royal Society aMathematical Physical and Engineering Sciences 2004, 362, 2477-2512.

3. Moon, I. K.; Lee, J.; Ruoff, R. S.; Lee, H., Reduced Graphene Oxide by Chemical Graphitization. Nature Communications 2010, 1.

4. Ferrari, A. C., Raman Spectroscopy of Graphene and Graphite: Disorder, ElectronPhonon Coupling, Doping and Nonadiabatic Effects. Solid State Communications 2007, 143, 4757. 